\title{
Nivel de conocimiento sobre métodos anticonceptivos y su uso en estudiantes de licenciatura
}

\author{
Pérez-Alegría Jasmín*, Castillo-Uribe Judith Alondra*, Vázquez-Guzmán Sharon*, Benítez-Cruz \\ Mabel*, Peza-Cruz Gabriela**
}

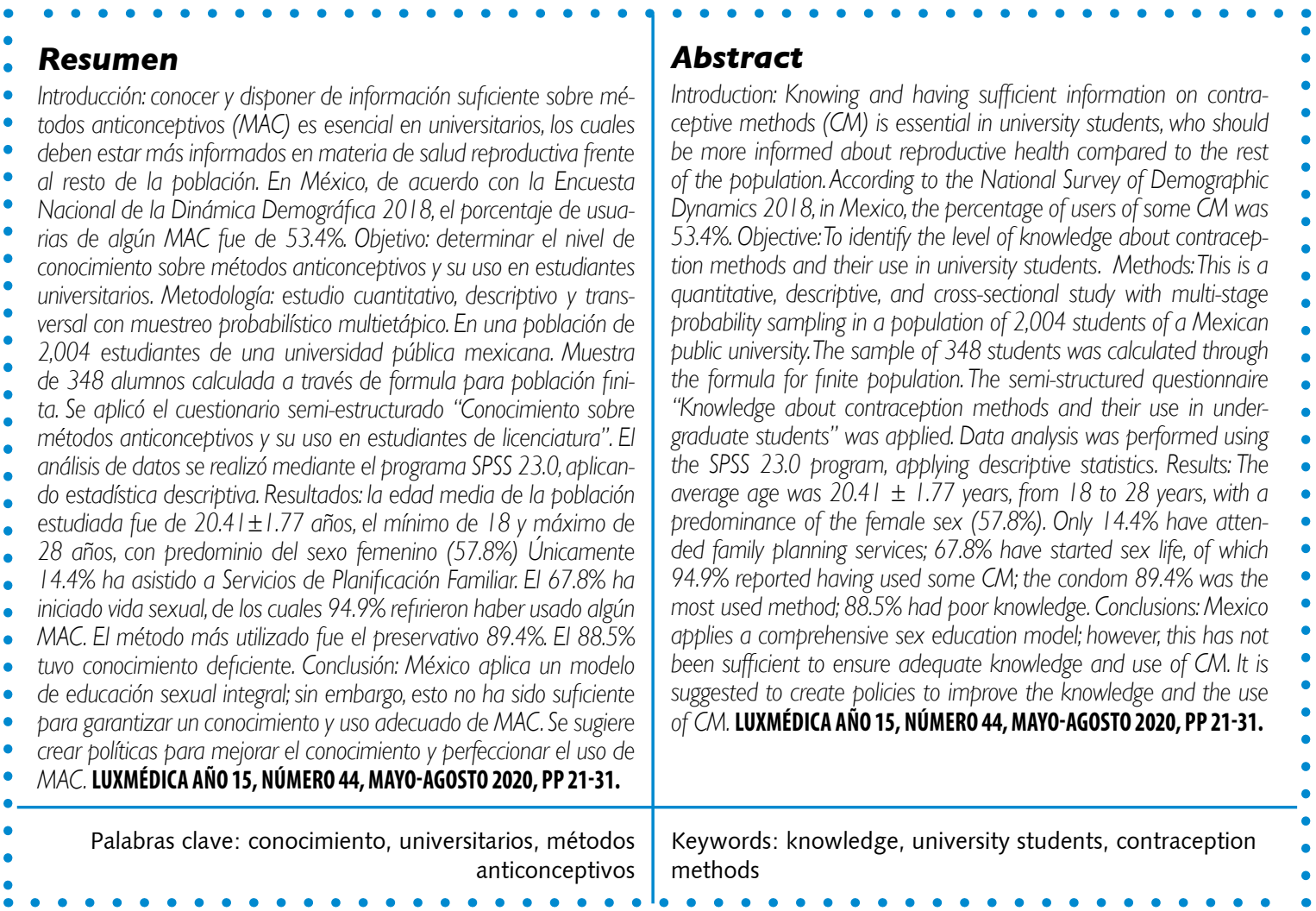

\footnotetext{
Pérez-Alegría Jasmín. Pasante de la Licenciatura en Enfermería. Facultad de Enfermería de la Universidad Autónoma de Querétaro. Campus San Juan del Rio, Qro. México. Correo electrónico jasperez.ale@gmail.com Orcid: 0000-0003-3533-9111

Castillo-Uribe Judith Alondra. Pasante de la Licenciatura en Enfermería. Facultad de Enfermería de la Universidad Autónoma de Querétaro. Campus San Juan del Rio, Qro. México. Correo electrónico julith12_21@hotmail.com Orcid: 0000-0001-5329-5349

* $\quad$ Vázquez-Guzmán Sharon Pasante de la Licenciatura en Enfermería. Facultad de Enfermería de la Universidad Autónoma de Querétaro. Campus San Juan del Rio, Qro. México. Correo electrónico sharon.v.guzman@gmail.com Orcid: 0000-0002-2361-4553

Benítez-Cruz Mabel Pasante de la Licenciatura en Enfermería. Facultad de Enfermería de la Universidad Autónoma de Querétaro. Campus San Juan del Rio, Oro. México. Correo electrónico mabelbc20897@hotmail.com Orcid 0000-0001-7796-6864

** Peza-Cruz Gabriela Maestra en Ciencias de la Enfermería. Docente de la Facultad de Enfermería de la Universidad Autónoma de Querétaro. Correo electrónico gabriela.peza@uaq.mx Orcid: 0000-0002-2995-345X
}

Fecha de recibido: 30 de octubre 2019

Fecha de aceptación: 4 de febrero 2020

Correspondencia: MCE Gabriela Peza Cruz. Facultad de Enfermería.

Universidad Autónoma de Querétaro, Campus San Juan del Río, Querétaro.

Av. Río Moctezuma \#249. Colonia San Cayetano, San Juan del Río,

Querétaro. México. CP. 76807. Teléfono:01 4421921200 extensión 5734.

Correo electrónico gabriela.peza@uaq.mx 


\section{Introducción}

Los métodos anticonceptivos (MAC) son procedimientos o productos que limitan la capacidad reproductiva de un individuo o pareja. ${ }^{1}$ Para la toma de decisiones reproductivas asertivas, es necesaria la educación sexual que es considerada actualmente como un derecho humano que hay que ejercer de manera informada, libre, satisfactoria, responsable y sin riesgos, de acuerdo con las preferencias y condiciones personales para lograr una mejor calidad de vida. $^{2}$ Es importante que los estudiantes universitarios refuercen el conocimiento sobre los MAC, debido a que diversos autores coinciden en que, dentro de este nivel educativo, se debe estar más informado en materia de salud reproductiva que el resto de la población, ${ }^{3,4}$ sobre todo porque México brinda un modelo integral para la educación sexual incluido desde el plan de estudios básico a medio superior. ${ }^{5}$

La falta de educación sexual y del conocimiento sobre el uso correcto de los MAC predispone a enfermedades de transmisión sexual, embarazos no deseados, embarazos a temprana edad y deserción escolar que, a pesar de los esfuerzos realizados, no se han logrado disminuir. En México, de acuerdo con la Encuesta Nacional de la Dinámica Demográfica 2018, el porcentaje de usuarias de algún MAC fue del $53.4 \% .^{6}$

El reto de otorgar la educación sexual a la población implica el acceso a información suficiente, clara, completa y veraz sobre métodos anticonceptivos para que las usuarias puedan seleccionar, de manera libre e informada, el MAC con base en necesidades, expectativas y condiciones de salud, así como garantizar la entrega del método seleccionado y dar seguimiento por parte del personal de salud. ${ }^{7}$

Recientemente, en el Programa de Acción Específico Planificación Familiar y Anticoncepción 2013-2018 se planteó dar a conocer las opciones disponibles para regular la fecundidad para facilitar la aceptación dentro de la población usuaria. Dicho programa ha dado a conocer que muchas mujeres en edad fértil conocen o han oído hablar por lo menos de un MAC. Aunque también se ha reportado que de 1997 al 2009, sólo hubo un incremento menor a 4 puntos porcentuales en prevalencia de uso de MAC y la menor tendencia se observó en el grupo de adolescentes, incrementándose hasta cerca del $80 \%$ después de los 35 años. $^{3}$

Los jóvenes universitarios representan un reto al no hacer uso de métodos anticonceptivos al pertenecer a un grupo etario caracterizado por conductas de riesgo como promiscuidad, intercambio de fluidos o actividad sexual bajo efecto del alcohol o estupefacientes. Esto los expone a embarazos no planeados, infecciones de transmisión sexual y a eventos negativos como la interrupción de la formación académica o incluso poner en peligro su vida. De ahí la importancia de conocer los métodos anticonceptivos y su uso adecuado. $^{8}$ 
Por otro lado, hay que recalcar que el nivel de conocimiento sobre el método anticonceptivo se relaciona de manera significativa con su uso ${ }^{9}$ por lo que resulta importante la adquisición del conocimiento como una herramienta indispensable para que se adopten decisiones libres e informadas en materia de anticoncepción. 2,10

Lo anterior resulta relevante al observar algunos cambios sociales, donde la vida de los jóvenes se ha tornado más liberal, al ver las relaciones sexuales de manera normal y como algo propio de la edad. ${ }^{11}$ Sin embargo, a pesar de que existe abundante información y acceso a los métodos anticonceptivos, diversos factores pueden influir para no utilizarlos o usarlos inadecuadamente. ${ }^{12} \mathrm{El}$ objetivo del presente estudio fue identificar el nivel de conocimiento y uso sobre métodos anticonceptivos en estudiantes de educación superior.

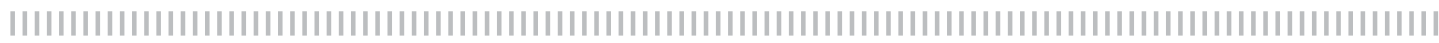

\section{Material y métodos}

Se realizó un estudio cuantitativo, descriptivo y transversal con muestreo probabilístico multietápico; se estableció como marco muestral las listas de alumnos proporcionadas por la institución. Se llevó a cabo la primera etapa estratificada proporcional por facultad (tabla 1) y una cantidad de grupos proporcional. Después, dentro de cada facultad, se aplicó el muestreo aleatorio simple para la selección de licenciaturas y semestres mediante la función aleatoria de
Excel. Para la selección de unidades de estudio, se empleó el software Lottery Random Number Generator (LottoGen) para generar números aleatorios con base en el número de alumnos.

El universo se conformó de 2,004 estudiantes de licenciatura inscritos en un campus perteneciente a una universidad pública. Participaron las facultades de contaduría y administración, psicología, derecho, ingeniería, enfermería, bellas artes y ciencias políticas y sociales.

\section{Tabla I}

Estimación de población y muestra de estudiantes de licenciatura por facultades de la Universidad Autónoma de Querétaro, campus San Juan del Río.

\begin{tabular}{|lccc|}
\hline & Población (N) & Muestra (n) \\
\hline Facultad & $\begin{array}{l}\text { Cantidad total } \\
\text { de estudiantes } \\
\text { de licenciatura }\end{array}$ & $\begin{array}{c}\text { Porcentaje } \\
\text { correspondiente } \\
\text { en relación con N }\end{array}$ & $\begin{array}{c}\text { Número de unidades } \\
\text { que se seleccionó de } \\
\text { cada facultad, según } \\
\text { el porcentaje determinado. }\end{array}$ \\
\hline Contaduría y Administración & 776 & 38.72 & 147 \\
\hline Psicología & 204 & 10.18 & 39 \\
\hline Derecho & 435 & 21.71 & 82 \\
\hline Ingeniería & 209 & 10.43 & 40 \\
\hline Enfermería & 176 & 8.78 & 33 \\
\hline Bellas Artes & 175 & 8.73 & 33 \\
\hline Ciencias Políticas y Sociales & 29 & 1.45 & 5 \\
\hline Total & 2004 & 100.00 & 379 \\
\hline
\end{tabular}

FUENTE: Universidad Autónoma de Querétaro. (2018). Unidad institucional de información. Recuperado de: http://planeacion.uaq.mx/uii/index.php/2017-2018 
Se consideró como criterio de inclusión que fueran estudiantes de licenciatura de la universidad y campus mencionado, inscritos en el periodo 2019-1, con edad entre 18 y 29 años y que aceptaran participar bajo consentimiento informado. Se excluyeron quienes no aceptaron participar en el estudio y aquellos ausentes al recolectar los datos. Finalmente, se consideró criterio de eliminación no contestar totalmente el instrumento, tener respuestas ilegibles y quienes decidieron abandonar el estudio. El tiempo aproximado de llenado fue de 15 minutos.

La muestra se calculó con una fórmula para población finita, se agregó $17.5 \%$ por índice de no respuesta en prueba piloto, resultando 379 alumnos. Para medir las variables de estudio, se utilizó un cuestionario semi-estructurado elaborado por Bastidas, Chávez, Orozco y Merchán en su estudio "Conocimientos y prácticas de métodos anticonceptivos en una población universitaria en el año 2010. Un estudio descriptivo", quienes mencionan que fue revisado por expertos en el área de salud sexual y reproductiva, y ajustada después de la prueba piloto.

Dicho cuestionario consta de 53 ítems repartidos en tres bloques: datos sociodemográficos, de conocimiento sobre anticoncepción y aspectos sobre las prácticas y uso de MAC. La aplicación del cuestionario se llevó a cabo previo consentimiento de los participantes. Para poder ser aplicado en el contexto mexicano se realizaron ajustes con base en la validez del constructo, además se realizó una prueba piloto en el campus.

Para la interpretación de la variable nivel de conocimiento se utilizó la suma de respuestas correctas con puntos de corte de una escala ordinal, identificando 4 niveles: excelente (18 a 20 respuestas), bueno (16 a 17 respuestas), regular (13 a 15 preguntas) y deficiente ( $<13$ preguntas).

Los datos obtenidos se capturaron en el programa SPSS versión 23, se aplicó estadística descriptiva: medidas de tendencia central y medidas de dispersión para las variables cuantitativas; para las variables cualitativas se calculó frecuencia y porcentaje. El nivel de conocimiento se consideró una variable cualitativa y se utilizó una escala de medición ordinal (excelente, bueno, regular y deficiente). Los resultados se presentan en tablas y gráficas.

La investigación se rigió por los principios bioéticos; beneficencia-no maleficencia, autonomía y justicia, además del Código de Núremberg, La Declaración de Helsinki, la Ley General de Salud (Artículo 100), el Reglamento de la Ley General de Salud; en Materia de Investigación para la salud (Artículos 13,14, 17,20, 21y 22) y a la Norma Oficial Mexicana NOM-012-SSA3-2012, que establece los criterios para la ejecución de proyectos de investigación para la salud en seres humanos. Además, se contó con el dictamen favorable del $\mathrm{H}$. Subcomité de Investigación de Pregrado y el Comité de Ética de la Facultad de Enfermería de la Universidad Autónoma de Querétaro.

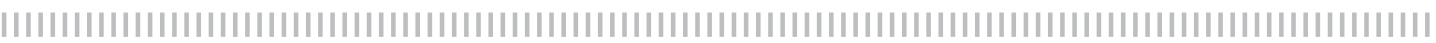

\section{Resultados}

Se excluyeron 31 alumnos que no cumplieron con criterios de selección integrando así una muestra de 348 estudiantes de una escuela de nivel superior, de los cuales $57.8 \%$ fueron mujeres y $42.2 \%$ fueron hombres, la media de edad fue $20.41 \pm 1.77$ años con mínimo de 18 y máximo de 28 años.

Con relación al estado civil, $96.3 \%$ se refirieron solteros y la religión católica fue predominante $(69.5 \%)$. El $87.1 \%$ es de nivel socioeconómico medio, $71.6 \%$ ha vivido en una zona urbana la mayor parte de su vida y el $82.5 \%$ vive en San Juan del Río.

En cuanto a la distribución de estudiantes por facultad, 39.7\% pertenece a Contaduría y Administración, 21.6\% a Derecho, 10.1\% Ingeniería, 9.5\% a Enfermería, 8.9\% a BeIlas Artes, $8.9 \%$ a Psicología y $1.4 \%$ a Ciencias Políticas y Sociales. Asimismo, el $69.5 \%$ 
no tiene estudios universitarios previos. En materia de salud, $83 \%$ se encuentra afiliado a Instituciones de Seguridad Social, 6\% no está asegurado, $4.6 \%$ pertenece al sector privado y $6.3 \%$ desconoce.

Por otro lado, el $85.6 \%$ nunca ha asistido a servicios de planificación familiar, por causas como: desconocer la existencia del servicio $(26.5 \%)$, no tener interés $(25.5 \%)$, no necesitarlo (21.5\%), no conocerlo (12.8\%), falta de tiempo (11.1\%), por pena o temor $(2.0 \%)$ y por falta de existencia del servicio en el lugar de residencia $(0.7 \%)$ (figura 1$)$.

Figura 1. Motivos de inasistencia al servicio de Planificación Familiar.

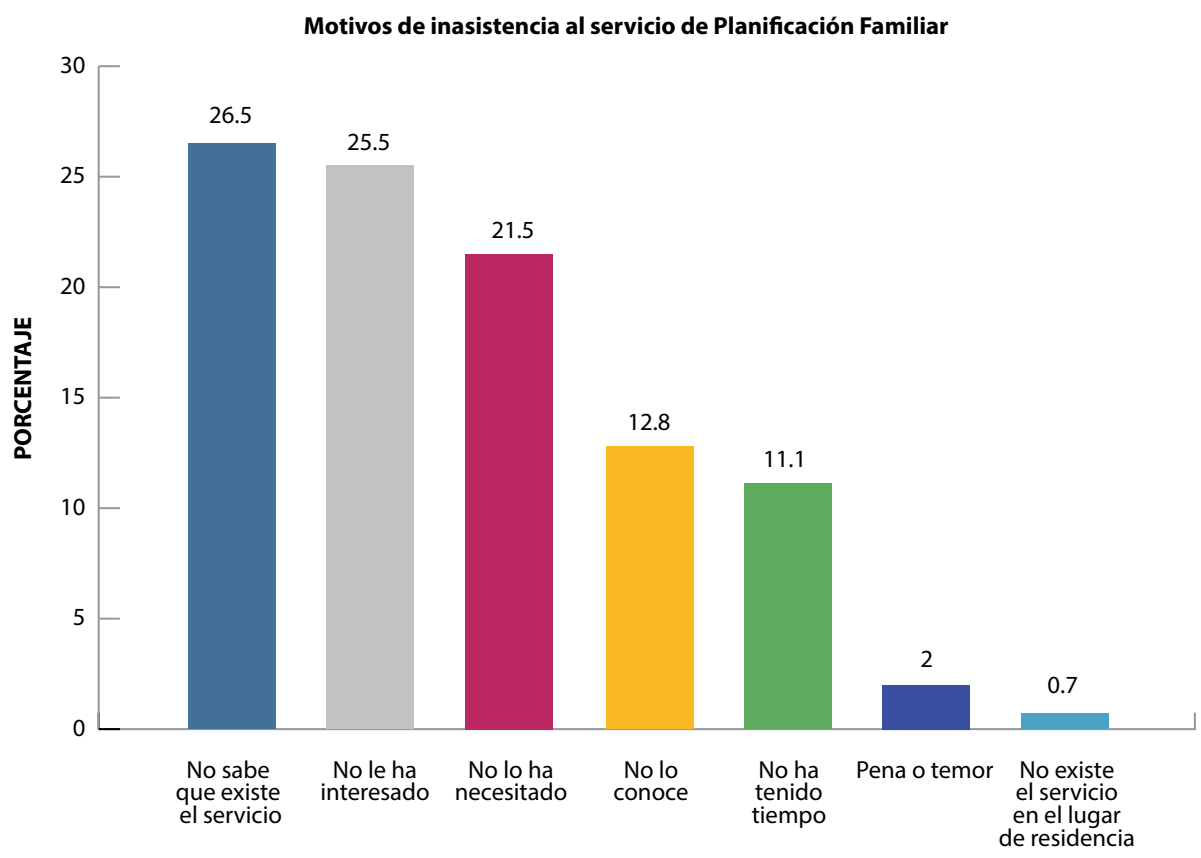

FUENTE: Nivel de conocimiento sobre métodos anticonceptivos y su uso en estudiantes de licenciatura.

2019-1 $\mathrm{n}=298$

Para la variable conocimiento, se determinó que el $88.5 \%$ tiene conocimiento deficiente, el $10.3 \%$ regular, el $1.1 \%$ tiene conocimiento bueno y ninguno reflejó un conocimiento excelente (tabla 2).

\section{Tabla 2}

Nivel de Conocimiento sobre Métodos Anticonceptivos en estudiantes de licenciatura

\begin{tabular}{|llccc|}
\hline \multicolumn{5}{c}{ Nivel de conocimiento } \\
\hline
\end{tabular}

FUENTE: Nivel de conocimiento sobre métodos anticonceptivos y su uso en estudiantes de licenciatura. 2019-1 
Además, en la variable Uso, el $94.9 \%$ de los estudiantes afirmó haber iniciado su vida sexual y usar algún método anticonceptivo. Se identificó en primer lugar $(89.4 \%)$ el preservativo, aunque sólo el $47.4 \%$ lo usa en todas las relaciones sexuales y el $53.1 \%$ al empezar la penetración vaginal. Respecto a la técnica de colocación, la medida de seguridad más empleada (32.7\%) fue comprobar la integridad del empaque y la menos contemplada $(4.8 \%)$ fue desenrollar el preservativo en su totalidad hasta la base del pene $y$, al terminar, desecharlo en un bote de basura (tabla 3 ).

\section{Tabla 3}

Técnica para la colocación del preservativo por la población en estudio.

\begin{tabular}{|c|c|c|c|c|}
\hline \multicolumn{5}{|c|}{ Técnica para colocación del preservativo } \\
\hline & \multicolumn{2}{|c|}{ No } & \multicolumn{2}{|c|}{ Sí } \\
\hline & $f$ & $\%$ & $f$ & $\%$ \\
\hline Comprobar que el condón no se encuentra caducado & 174 & 82.5 & 37 & 17.5 \\
\hline Comprobar que se encuentre el empaque en óptimas condiciones & 142 & 67.3 & 69 & 32.7 \\
\hline Abrir con cuidado de no romperlo con uñas, dientes o anillos & 170 & 80.6 & 41 & 19.4 \\
\hline $\begin{array}{l}\text { Comprobar que el condón esté del lado correcto para facilitar el } \\
\text { deslizamiento }\end{array}$ & 200 & 94.8 & 11 & 5.2 \\
\hline $\begin{array}{l}\text { Colocar el preservativo en la punta del pene, presionando la } \\
\text { punta del condón entre el dedo pulgar e índice, para evitar que } \\
\text { queden burbujas de aire }\end{array}$ & 184 & 87.2 & 27 & 12.8 \\
\hline $\begin{array}{l}\text { Desenrollar en su totalidad el preservativo con el aro hacia afuera } \\
\text { en el pene (hasta la base) }\end{array}$ & 206 & 97.6 & 5 & 2.4 \\
\hline $\begin{array}{l}\text { Retirar el condón con un pedazo de papel y desechar en un bote } \\
\text { de basura }\end{array}$ & 206 & 97.6 & 5 & 2.4 \\
\hline
\end{tabular}

FUENTE: Nivel de conocimiento sobre métodos anticonceptivos y su uso en estudiantes de licenciatura. 2019-1 $\quad n=211$

De manera que el $53.1 \%$ realiza un uso parcialmente correcto al describir de 1 a 3 precauciones al colocar el preservativo. En cambio, el $44.5 \%$ no escribió ninguna pre- caución empleada, reflejando un uso incorrecto y sólo el $2.4 \%$ escribió más de tres medidas, manifestando un uso correcto de acuerdo con la NOM-005 (figura 2).

Figura 2. Uso del preservativo en estudiantes de licenciatura. $n=211$

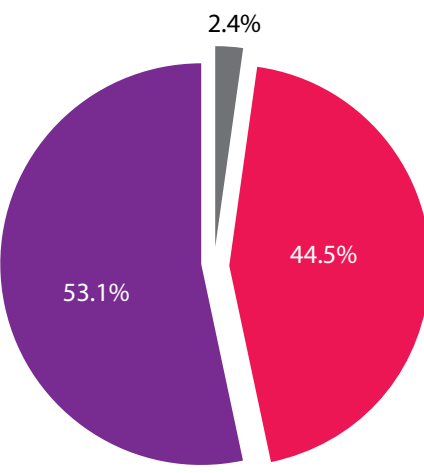

Incorrecto
FUENTE: Nivel de conocimiento sobre métodos anticonceptivos y su uso en estudiantes de licenciatura. 2019-1 
No obstante, el segundo método más utilizado fue el coito interrumpido (23.3\%), seguido de las pastillas anticonceptivas $(15.3 \%)$, las cuales eran tomadas correctamente por el $55.6 \%$. El cuarto método más utilizado fue el método del calendario $(12.3 \%)$, posteriormente las inyecciones anticonceptivas, las cuales eran utilizadas en un $3 \%$ de los estudiantes y aplicadas adecuadamente en tiempo y forma por el $85.7 \%$ de los estudiantes o sus parejas.

Los métodos anticonceptivos de menor uso fueron el DIU (2.5\%), parche anticonceptivo (1.7\%), espermicidas (1.3\%), implante subdérmico $(1.3 \%)$ y la vasectomía $(0.4 \%)$ (tabla 4$).$

\section{Tabla 4}

Métodos anticonceptivos utilizados por los estudiantes de licenciatura.

\begin{tabular}{|lcccc|}
\hline \multicolumn{1}{c}{ Método anticonceptivo } & \multicolumn{3}{c}{} \\
\hline & \multicolumn{2}{c}{ No } & \multicolumn{2}{c}{$\mathrm{Si}$} \\
\hline & $\mathrm{f}$ & $\%$ & $\mathrm{f}$ & $\%$ \\
\hline Preservativo & 25 & 10.6 & 211 & 89.4 \\
\hline Coito interrumpido & 181 & 76.7 & 55 & 23.3 \\
\hline Pastillas Anticonceptivas & 200 & 84.7 & 36 & 15.3 \\
\hline Método del calendario o ritmo & 207 & 87.7 & 29 & 12.3 \\
\hline Inyecciones anticonceptivas & 229 & 97.0 & 7 & 3.0 \\
\hline Dispositivo Intrauterino & 230 & 97.5 & 6 & 2.5 \\
\hline Parche Anticonceptivo & 232 & 98.3 & 4 & 1.7 \\
\hline Espermicidas & 233 & 98.7 & 3 & 1.3 \\
\hline Implante Subdérmico & 233 & 98.7 & 3 & 1.3 \\
\hline Vasectomía & 235 & 99.6 & 1 & 0.4 \\
\hline
\end{tabular}

FUENTE: Nivel de conocimiento sobre métodos anticonceptivos y su uso en estudiantes de licenciatura. 2019-1 $n=236$

Respecto a la anticoncepción postcoital, con la pastilla de emergencia (PAE), el $69.5 \%$ de los estudiantes que iniciaron vida sexual la había utilizado alguna vez en su vida. De ese porcentaje, el $29.3 \%$ la utilizó hace más de un año, $46.3 \%$ sólo una vez en el último año, $14 \%$ dos veces y $10.4 \%$ tres o más veces en los últimos 12 meses (tabla 5).

\section{Tabla 5}

Frecuencia de uso de la Pastilla Anticonceptiva de Emergencia en el último año. Uso de PAE en el último año

\begin{tabular}{|l|c|c|}
\hline & $f$ & $\%$ \\
\hline Ninguna & 48 & 29.3 \\
\hline Sólo una vez & 76 & 46.3 \\
\hline Dos veces & 23 & 14.0 \\
\hline Tres veces o más & 17 & 10.4 \\
\hline
\end{tabular}

FUENTE: Nivel de conocimiento sobre métodos anticonceptivos y su uso en estudiantes de licenciatura. 2019-1 n=164 
En cuanto a la fuente de obtención de información, la principal fue el colegio (68.4\%), es decir, la educación básica y media superior, seguida de los medios de comunicación $(59.2 \%)$, servicios de salud en general $(55.7 \%)$, familiares $(52.6 \%)$, universidad $(43.1 \%)$, amigos $(42.5 \%)$, pareja $(29.9 \%$ ) y $11.5 \%$ ENSAIN (Clínica de la Facultad de Enfermería).

| | | | | | | | | | | | | | | | | | | | | | | | | | | | | | | | | | | | | | | | | | | | | | | | | | | | | | | | | | | | | | | | | | | | | | | | | | | | | | | | | | | | | | | | | | | | | | | | | | | | | | | | | | | |

\section{Discusión}

De acuerdo con los resultados obtenidos, se puede constatar que la educación sexual es deficiente o nula entre los universitarios y la institución educativa no figura como eje formativo en esta materia. La mayoría de los participantes de la investigación clasifican como adultos jóvenes, en los cuales la edad y el sexo fueron similares a poblaciones universitarias estudiadas en otras investigaciones. Más de la mitad de los universitarios $(69.5 \%)$ profesa la religión católica, resultados que concuerdan con Covarrubias. ${ }^{13}$

En cuanto a características sobre salud sexual y reproductiva, el $67.8 \%$ de los estudiantes ha iniciado relaciones sexuales, reportando haber tenido entre 2 y 3 parejas sexuales, número similar al descrito por otros autores. ${ }^{13}$ Sólo el $2 \%$ tiene hijos, los cuales fueron no planeados en el $71.4 \%$ de los casos. La edad de la primera relación sexual (17.12 \pm 1.81 años) concordó con la edad de inicio de vida sexual del país. Según el Consejo Nacional de Población, el inicio de vida sexual se vincula con la trayectoria reproductiva, principalmente al número de hijos y la edad del primer embarazo. ${ }^{14}$

En cuanto a la variable de conocimiento los resultados obtenidos fueron semejantes a los expuestos por Bastidas ${ }^{5}$, donde el $88 \%$ de estudiantes tenía conocimientos deficientes, el $10.7 \%$ conocimiento regular, sólo el $1.3 \%$ poseía un conocimiento bueno y ningún participante alcanzó el excelente, ambas poblaciones estudiadas cursaban la educación superior.

En el estudio Nivel de conocimientos sobre métodos anticonceptivos entre es- tudiantes de Medicina Humana de $1^{\circ}$ a $3^{\circ}$ año realizado en Perú, se menciona que la escuela no figura como un eje formativo en materia de sexualidad, ${ }^{4}$ lo cual difiere con los resultados de esta investigación, donde el $68.4 \%$ reconoció a la educación básica y media superior como principal fuente de información sobre métodos anticonceptivos. Sin embargo, el $43.1 \%$ considera que la universidad no es de las primeras fuentes informativas que utiliza: a pesar de que el campus cuenta con una Clínica Universitaria ENSAIN, únicamente el $11.5 \%$ de los estudiantes la reconoció como fuente de información.

Respecto a la utilización, los universitarios desconocen el uso adecuado de los MAC, impidiendo el acceso al máximo porcentaje de efectividad, ${ }^{15}$ y aunque resulta positivo que el $94.9 \%$ afirmara hacer uso de algún MAC, sería coherente poseer de manera paralela bases teóricas para realizar un uso correcto, puesto que de eso depende la eficacia del método, no obstante, esto no se vio reflejado en el estudio. Por lo tanto, se coincide con Navalón \& Ruiz, refiriendo que existe diferencia entre el conocimiento del universitario y la conducta que asume. ${ }^{16}$

Un dato poco novedoso fue encontrar en la mayoría (89.4\%) el uso del preservativo masculino, dando como principales motivos el fácil acceso, el bajo costo, no tener efectos secundarios, ni alteración de la sexualidad o problemas futuros de fecundidad. Es decir, existe preferencia por el condón sobre otros métodos y es reconocido como la forma más confiable de evitar una infección de transmisión sexual. 5,13 
En cuanto al uso, se sabe que existe una tendencia de creencias asociadas al preservativo y a la satisfacción sexual. Esto dificulta el uso y ocasiona una disminución paulatinamente a partir de la primera relación sexual. ${ }^{17}$ En este sentido, se encontró que sólo el $22.3 \%$ de los universitarios emplea el preservativo en todas las relaciones sexuales y al empezar la penetración vaginal, lo que deja al porcentaje restante con riesgo de un embarazo no planeado o de contraer una infección de transmisión sexual.

El embarazo en universitarias abre una línea de investigación, puesto que uno de cada cuatro embarazos entre adolescentes no fue planeado y uno de cada diez tampoco fue deseado. ${ }^{14}$ Además se notifica que las universitarias y madres adolescentes viven su maternidad como un momento de crisis en el aspecto psicosocial, debido a los cambios en su proyecto de vida, que desestabilizan las relaciones familiares y académicas, sobre todo por la relación entre el embarazo universitario y deserción escolar. ${ }^{17-19}$

Además del preservativo, resaltaron el coito interrumpido $(23.3 \%)$, las pastillas anticonceptivas $(15.3 \%)$, el método del calendario $(12.3 \%)$, las inyecciones anticonceptivas $(3.0 \%)$, el DIU $(2.5 \%)$, el parche anticonceptivo (1.7\%), los espermicidas $(1.3 \%)$, el implante subdérmico $(1.3 \%)$ y la vasectomía $(0.4 \%)$.

Respecto a la anticoncepción postcoital, el $69.5 \%$ de los estudiantes refieren haberla utilizado alguna vez en su vida. De ese porcentaje, el $24.4 \%$ la ocupó más de dos veces en el último año, lo cual es alarmante, debido a que algunos estudios identifican a más del $80 \%$ de hombres y mujeres considerando a la pastilla de emergencia como un método de planificación familiar. ${ }^{19,20}$ Lo anterior obliga a cuestionar sobre el nivel de información con el que cuentan los universitarios acerca de esta alternativa no recomendada para un uso rutinario.

Al comparar los resultados de investigaciones similares con los obtenidos en este estudio, se esperaría que, al tratarse de universitarios, su conocimiento fuera de mejor nivel, por el status frente al resto de la población. Sin embargo, se aprecia que, a pesar de ser una población localizada en un medio urbano y que cuenta con formación universitaria, es limitado el uso personal de MAC como una estrategia de autocuidado eficaz en lo que respecta a la salud sexual y reproductiva.

\section{Recomendaciones}

México imparte un modelo integral de educación sexual desde el grado básico hasta el grado medio superior dentro de los planes de estudio y se ha demostrado que esto no ha garantizado el conocimiento y uso adecuado de los MAC. Por ello, se propone como área de oportunidad para la Clínica Universitaria, la reestructuración de las políticas y estrategias, dirigidas a los universitarios para aumentar el nivel de conocimiento y uso de MAC. Esto garantizaría el uso correcto y el acceso a una educación integral mediante herramientas teóricas y prácticas necesarias para que los universitarios puedan ejercer plenamente su sexualidad de manera sana y responsable.

Además, es necesario profundizar en estudios sobre temas sexuales que aborden la percepción del universitario y las estrategias a implementar para posicionar a la universidad como principal fuente de información sobre métodos anticonceptivos. De igual forma, se abren diversas líneas de investigación que pueden proporcionar más datos sobre prácticas sexuales de riesgo entre la población universitaria. 


\section{Conclusiones}

La presente investigación mostró que casi 9 de cada 10 universitarios tienen conocimiento deficiente sobre métodos anticonceptivos. Éstos no consideran a la universidad como eje formativo en salud sexual, situación preocupante por la postura que debería corresponder a este nivel de preparación académica. Alarma encontrar un alto uso de anticonceptivos y un bajo conocimiento o un uso incorrecto. El método anticonceptivo más recurrente fue el condón masculino y se identificó un mal empleo de la anticoncepción de emergencia.

Por lo tanto, es necesario mejorar la calidad de información que poseen los universitarios sobre el uso adecuado de MAC debido a que el $94.9 \%$ los utiliza influyendo así positivamente en la salud sexual y reproductiva.

Este estudio es importante porque muestra los resultados representativos del campus universitario que, al ser un reflejo del conocimiento que poseen los universitarios, permite tener una visión ampliada de la realidad de los jóvenes frente a su sexualidad, de su conocimiento de métodos anticonceptivos y su uso. Con base en lo anterior, será posible desarrollar espacios de difusión y divulgación de resultados con la finalidad de buscar la generación de estrategias que garanticen un mayor impacto en el área de educación, prevención y promoción en salud sexual y reproductiva de los jóvenes universitarios.

\section{Bibliografía}

1. Gamboa MG, Gutiérrez SM. Métodos anticonceptivos. Glosario, antecedentes, marco jurídico, políticas públicas y recomendaciones internacionales. [Internet]. 2015 Julio: [citado 28 de agosto de 2019]; 1-103. Disponible en: http://www.cfnavarra.es/salud/anales/textos/vol26/n3/revis2a.html

2. Secretaría de Salud. Programa de Acción Específico (PAE). Planificación Familiar y Anticoncepción 20132018. [Internet] Agosto 2015: [citado 28 de agosto de 2019]: 1-41; Disponible en: https://www.gob. $\mathrm{mx} /$ salud/acciones-y-programas/programa-de-accion-especifico-planificacion-familiar-y-anticoncepcion-2013-2018-10070

3. Méjico MJJ, Melgar GMF, Mendoza ML, Salinas CW. Nivel de conocimiento sobre métodos anticonceptivos entre estudiantes de Medicina Humana de $1^{\circ}$ a $3^{\circ}$ año. Rev Horiz Med. [Internet]. 2012 Septiembre [citado 28 de agosto de 2019] ; 12(3):35-41: Disponible en: http://www.horizontemedicina.usmp.edu. pe/index.php/horizontemed/article/view/129/127

4. Bastidas-Sánchez BE, Chávez M del S, Orozco-Chamorro CM, Merchán-Galvis Ángela M. Conocimientos y prácticas de métodos anticonceptivos en una población universitaria en el año 2010. un estudio descriptivo. Rev. Fac. Cienc. Salud Univ. Cauca [Internet]. 1 de agosto de 2014 [citado 28 de agosto de 2019];16(2):10-16. Disponible en: https://revistas. unicauca.edu.co/index.php/rfcs/article/view/27

5. Secretaria de educación pública. Agenda sectorial para la educación integral en sexualidad con énfasis en la prevención del embarazo en adolescentes. Avances. Líneas de acción en la ANAPEA. [Internet] 2016. México. Oficialía Mayor. Área igualdad de género [citado 28 de agosto de 2019]:1-47. Disponible en: https://www.gob.mx/cms/uploads/ attachment/file/230821/8_Acciones_SEP Dra Silvia_Ramirez.pdf

6. Instituto Nacional de Estadística y Geografía (INEGI). Encuesta Nacional de la Dinámica Demográfica 2018 [Internet]. 2019: [citado 26 de agosto de 2019]; 3-26 Disponible en: https://www.inegi.org.mx/programas/enadid/2018/

7. Instituto Mexicano del Seguro Social (IMSS). Planificación Familiar [Internet] 2017. [citado 23 de noviembre de 2019] Disponible en: http://www.imss. gob.mx/salud-en-linea/planificacion-familiar

8. Forcada MP, Pacheco MAS, Pahua ME, Palacios RPP, Todd NE, Pulido RMA. Conducta sexual de riesgo en estudiantes universitarios: factores de riesgo y protección. Revista Intercontinental de Psicología y Educación [Internet]. 2013. [citado 28 de agosto de 2019]:15(1):23-46. Disponible en: http://www.redalyc.org/articulo.oa?id =80225697003

9. Del Pino D. Nivel de conocimiento y uso de métodos anticonceptivos en madres adolescentes del Centro de Salud Chorrillos I Gustavo Lanatta Luján febreroabril 2015 [tesis] Lima-Perú: Universidad Nacional Mayor de san Marcos; 2016

10. Rodríguez TE, Orozco K, Gil LM, Mosquera N. Uso y conocimiento de métodos anticonceptivos en estu- 
diantes de décimo y undécimo grado, Puerto Tejada- Cauca. Ciencia \& Salud. [Internet] 2015: [citado 28 de agosto de 2019]: 3(12):37-43. Disponible en: https://pdfs.semanticscholar.org/45ae/743aea83f5 e6dc251588165f3fc1c11cae2f.pdf

11. Chanamé-Quezada F, Coronado-García M, MejíaVásquez $K$, Palacios-Apaéstegui $M$, Revilla-Domínguez S, Díaz-Vélez C. Características del uso de métodos anticonceptivos en estudiantes de la universidad de Chiclayo, Perú. Agosto - octubre 2016. RHCS [Internet]. 11 mayo 2016 [citado 28 Agosto 2019];2(4):272-81. Disponible en: http:// www.uhsalud.com/index.php/revhispano/article/ view/211

12. Mondragón-Rosales LA, Arteaga-Garcia RJ, Islas-Toris $\mathrm{P}$, Sanchez-Nuncio HR. Conocimientos, actitudes y prácticas sobre los métodos anticonceptivos en estudiantes de medicina. Revista de la Escuela de Medicina "Dr. José Sierra Flores" [Internet]. 2014 [citado 28 Agosto 2019]; 28(2), 71-80 Disponible en: http://www.une.edu.mx/ Resources/RevistaMedicina/2014/2014-02/3-Conocimientos, \% 20actitudes $\% 20 y \% 20$ prácticas $\% 20$ sobre $\% 20$ los $\%$ 20métodos $\% 20$ anticonceptivos $\% 20$ en\% 20estudiantes\% 20de\% 20medicina.pdf

13. Covarrubias E, Ramírez R, de Jesús E, Rivas J, Rivero L. Utilización de métodos anticonceptivos en estudiantes de enfermería. Investigación en Enfermería: Imagen y Desarrollo [Internet]. 2016 [citado 25 noviembre 2019];18(1):31-43. Recuperado de: https:// www.redalyc.org/articulo.oa?id $=145243501003$

14. Consejo Nacional de Población. Situación de la Salud Sexual y Reproductiva. República Mexicana [internet]. México: Dirección de Estudios Sociodemográficos; 2016 [citado 25 de noviembre de 2019]. Disponible en: https://www.gob.mx/cms/uploads/ attachment/file/237216/Cuadernillo_SSR_RM.pdf

15. Acevedo G, Ramírez N, Cardona J, Oliveros C.,
Conocimiento y uso de método anticonceptivo de emergencia en estudiantes universitarios, Pereira, Colombia. Univ. Salud. [Internet]. 2019 [citado 25 noviembre 2019];21(2): 159-165. Disponible en: http://www.scielo.org.co/scielo.php?pid=S012471 072019000200159\&script $=$ sci abstract\&tlng $=e s$.

16. Navalón, A. \& Ruiz, R. Análisis sociológico de los factores de riesgo sexual en la población universitaria. Un estudio comparativo. Departamento de Sociología I. Univesidad de Alicante, España. Index Enferm. [Internet] 2015 [citado 28 agosto 2019]; 24(1-2):40-43

17. Abril-Valdez E., Román-Pérez R., Cubillas-Rodríguez M.J., Domínguez-Ibáñez S.E. Creencias sobre el uso del condón en una población universitaria. Revista Científica Multidisciplinaria de Prospectiva [Internet]. 2018 [citado 26 noviembre 2019]; 25(3):1-24. Disponible en: https://www.redalyc.org/jatsRepo/104/10455646003/index.html

18. Moreno B, Arrellano G, Colin M, Lomas A, Sánchez L. Deserción de Estudiantes Universitarios por Embarazo. Rev. CuidArte "El Arte del Cuidado" [Internet]. 2013. [citado 25 noviembre 2019] 2(4):30-37. Disponible en: http://salutsexual.sidastudi.org/resources/inmagic-img/DD38409.pdf

19. Mori?n-Jua?rez A, Trevin?o-Alani?s M, Rivera-Silva G. Estudiantes universitarias embarazadas. [Internet] Rev Med Inst Mex Seguro Soc. 2019[Consultado el 26 noviembre 2019];57(1):4-5. Disponible en: http://revistamedica.imss.gob.mx/editorial/index. $\mathrm{php} / \mathrm{revista}$ medica/article/download/2622/3587

20. Tapia-Curiel A, Villaseñor-Farías $M$, Nuño-Gutiérrez BL. Conocimientos y actitudes hacia el uso de la anticoncepción de emergencia en jóvenes universitarios. Revista Médica del Instituto Mexicano del Seguro Social. [Internet] 2008. [citado 28 agosto 2019];46(1):33-41. Recuperado de: http://www. redalyc.org/pdf/4577/457745519006.pdf 